\title{
The embedded star clusters in the nebulae NGC 2327 and BRC 27 in Canis Majoris R1
}

\author{
J. B. Soares and E. Bica \\ Universidade Federal do Rio Grande do Sul, IF, CP 15051, Porto Alegre 91501-970, RS, Brazil
}

Received 11 February 2002 / Accepted 21 March 2002

\begin{abstract}
We employed $J, H$ and $K_{\mathrm{s}}$ photometry from the 2MASS Catalogue to study the embedded star clusters in the nebulae NGC 2327 and BRC 27 locate in the molecular cloud Canis Majoris R1. We made use of colourcolour and colour-magnitudes diagrams of the sample objects in conjunction with those of the Trapezium cluster which was used as a template, together with theoretical isochrones. The fundamental parameters were derived for the clusters. The distances found for NGC 2327 and BRC 27 were $1.2 \mathrm{kpc}$ which are compatible with previous distances for the complex. The estimated ages are based mostly on the number of stars with anomalous colours, supposedly of types Herbig AeBe and T Tauri. Both embedded clusters have ages of $\approx 1.5$ Myr. The results suggest the birth of a physical pair of star clusters in CMaR1.
\end{abstract}

Key words. Galaxy: open clusters and stellar associations: individual: NGC 2327, BRC 27

\section{Introduction}

The sensitivity increase of IR detectors provided new opportunities to observe and analyse the stellar content in molecular clouds. Many studies of these regions revealed the presence of embedded clusters (e.g. Hodapp 1994), with stars presenting IR excess which are characteristic of very young objects. Colour-colour diagrams are a useful tool to deal with very young objects in the premain-sequence (PMS) stage (Carpenter et al. 1993). This method enables detections of $K$ or $K_{\mathrm{s}}$ band excesses, which originate in dust disks or envelopes of contracting objects. Large reddening values are obtained in these regions as compared to surrounding field stars.

The CMaR1 star formation region was first identified as a group of stars embedded in reflection nebulosity by van den Bergh (1966). In the same area Clariá (1974a, b) identified an OB stellar association which appears to be a slightly evolved component of the star forming complex. The association CMaR1 is characterized by the occurrence of several Herbig AeBe stars. Clariá (1974b) and Shevchenko (1999) have found a distance of $\approx 1.15 \mathrm{kpc}$ and $\approx 1.05 \mathrm{kpc}$ to the association, respectively. The star $\mathrm{LkH} \alpha 221$ with spectral type B5 (Clariá 1974a) is embedded in the nebula NGC 2327. Although several studies have analysed this star, none focused on the hypothesis of

Send offprint requests to: J. B. Soares,

e-mail: jules@if.ufrgs.br the existence of a related surrounding star cluster or stellar group. We also study BRC 27 which is a bright-rimmed cloud (BRC) in the direction of CMaR1 (Sugitani et al. 1991). Bright-rimmed clouds have been considered potential sites of star formation due to the gas compression by ionization shock fronts. A young star cluster related to BRC 27 has been reported by Sugitani et al. (1995) by means of infrared imaging.

The 2MASS (2 Micron All Sky Survey) Atlas and Catalogue are the result of an all-sky near infrared survey (Skrutskie et al. 1997). The effective wavelengths are $1.25 \mu \mathrm{m}(J), 1.65 \mu \mathrm{m}(H)$ and $2.17 \mu \mathrm{m}\left(K_{\mathrm{s}}\right)$. The $K_{\mathrm{s}}$ band is thus located slightly shortwards of the $K$ band. The data so far available (second incremental release) can be obtained in the web interface http://irsa.ipac.caltech.edu, and they will be employed in the regions of NGC 2327 and BRC 27.

In the present study we analyse the stellar content of the nebulae NGC 2327 and BRC 27 by means of colourcolour and colour-magnitude diagrams (CMD) using the 2MASS Point Source Catalog. In particular we address the possibility of an embedded star cluster surrounding the star LkH $\alpha 221$. In Sect. 2 we describe the objects based on 2MASS imaging. In Sect. 3 we present the 2MASS photometric extractions in the bands $J H K_{\mathrm{s}}$. In Sect. 4 we explain the data analysis methods, including the diagrams of a template embedded cluster. In Sects. 5 and 6 we discuss the photometric diagrams of the embedded objects 


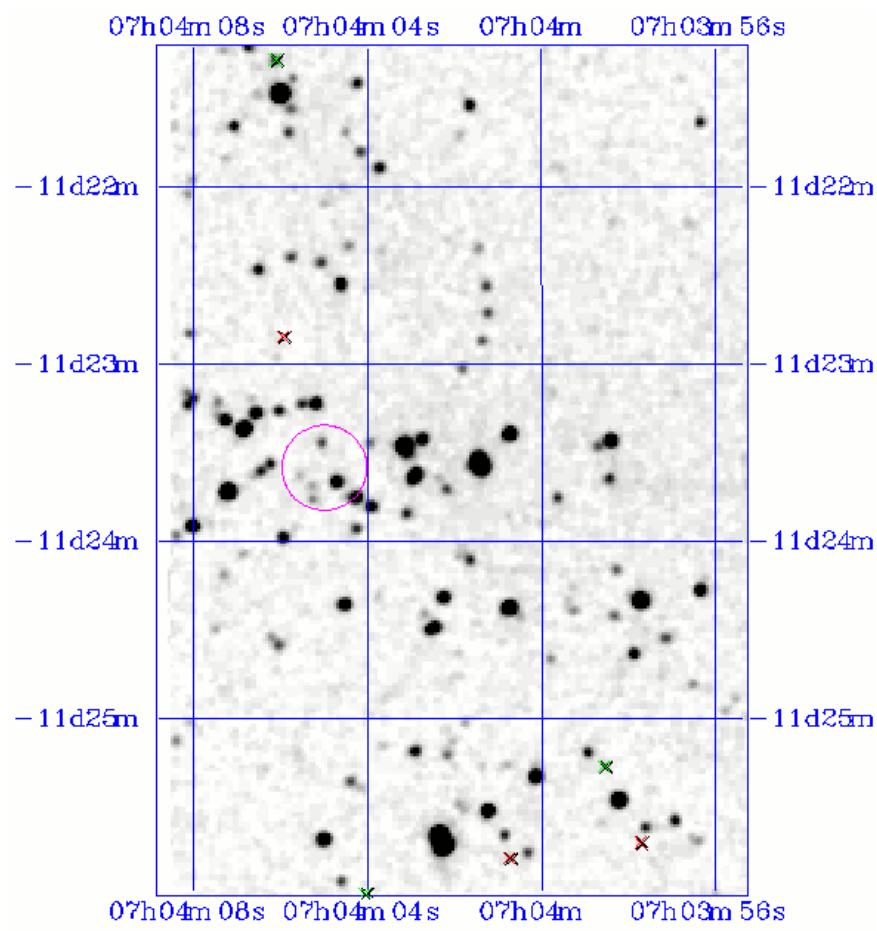

Fig. 1. $K_{\mathrm{s}}$ band 2MASS image of the BRC 27 region. Open circle indicates the object central part. Crosses indicate instrumental artifacts.

in NGC 2327 and BRC 27, respectively. Finally, in Sect. 7 the concluding remarks of this work are presented.

\section{The objects}

The stellar enhancement in BRC 27 is located at J2000.0 $\alpha=7^{\mathrm{h}} 04^{\mathrm{m}} 04^{\mathrm{s}} \delta=-11^{\circ} 23^{\prime} 40^{\prime \prime} \quad\left(l=224.51, b=-2^{\circ} .41\right)$. A $K_{\mathrm{S}}$ band image of the region is presented in Fig. 1, which was obtained from the 2MASS Atlas. The cluster or stellar group is elongated with dimensions $\approx 3.5^{\prime} \times 2^{\prime}$.

In Fig. 2 is shown the $K_{\mathrm{s}}$ image of the NGC 2327 region, where a stellar concentration stands out from the sourrounding field. The cluster or stellar group in NGC 2327 is located at $\alpha=7^{\mathrm{h}} 04^{\mathrm{m}} 08^{\mathrm{s}} \delta=-11^{\circ} 18^{\prime} 57^{\prime \prime}(l=$ $224^{\circ} .44, b=-2^{\circ} .36$ ) and its angular diameter is $\approx 2^{\prime}$.

An $R$ band Digitized Sky Survey image in the area of both objects is given in Fig. 3, which shows the importance of dust absorption and emission. NGC 2327 is prominent, with a refletion component and some gas emission expected, since a B5 star is embedded. The bright rim (Sugitani et al. 1991) of BRC 27 is evident. Extinction estimates in the directions of the 2 objects can be obtained from Schlegel et al. (1998): $A_{V}=8.2$ for BRC 27 and $A_{V}=11.9$ for NGC 2327. Owing to strong extinction the stellar content of the 2 nebulae requires IR photometry for detailed studies.

In Fig. 4 is shown an IRAS image in the $12 \mu \mathrm{m}$ band of CMaR1. This band is particularly sensitive to warm dust, and in turn to the possible presence of star formation. The present 2 objects occupy the same blob in a nearly central position in the dust/molecular cloud. However, the cloud

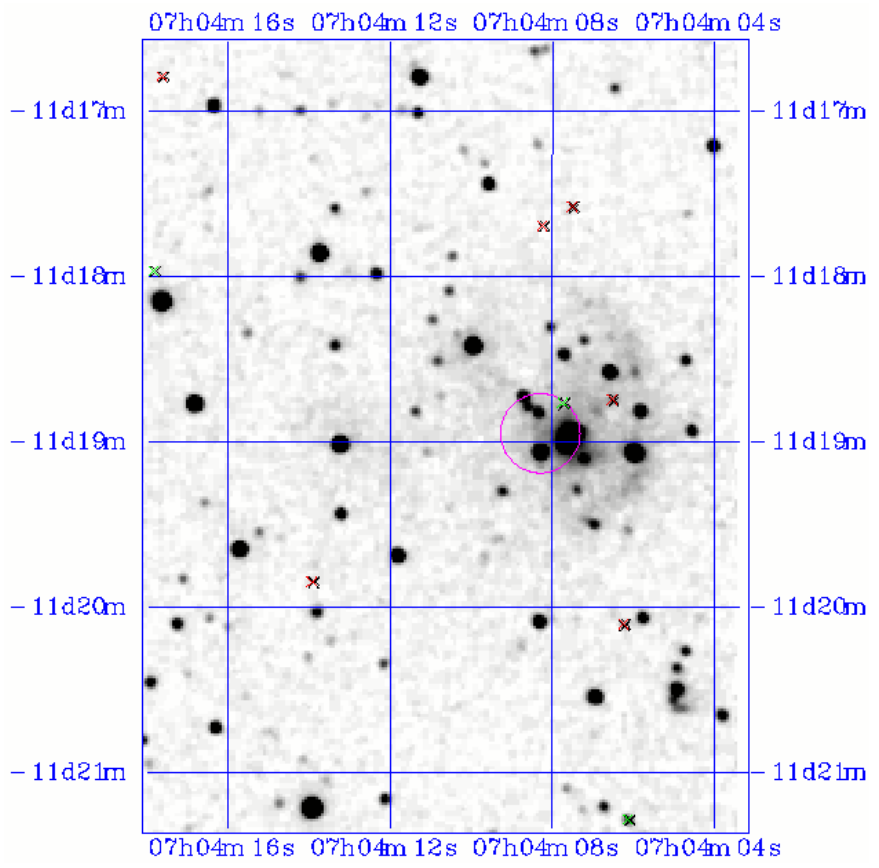

Fig. 2. Same as Fig. 1 for the NGC 2327 region.

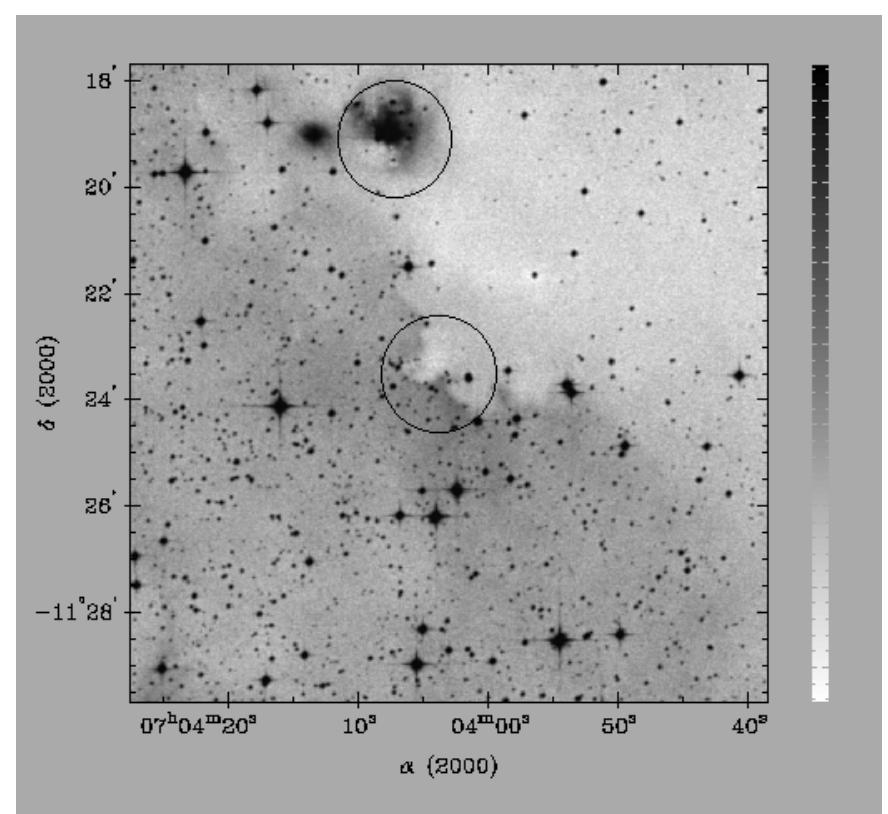

Fig. 3. Optical $R$ image from the Digitized Sky Survey, including the nebulae BRC 27 (within lower circle) and NGC 2327 (upper circle).

appears to have several cores, probably caused by a shock front. Indeed, the present blob is part of crescent-shaped structure characterizing the southern part of CMaR1.

\section{IR photometry}

We employed the Gator tool for 2MASS Point Source Catalog extractions. An exctraction table provides for each star besides the $J, H$ and $K_{\mathrm{s}}$ magnitudes, the three 


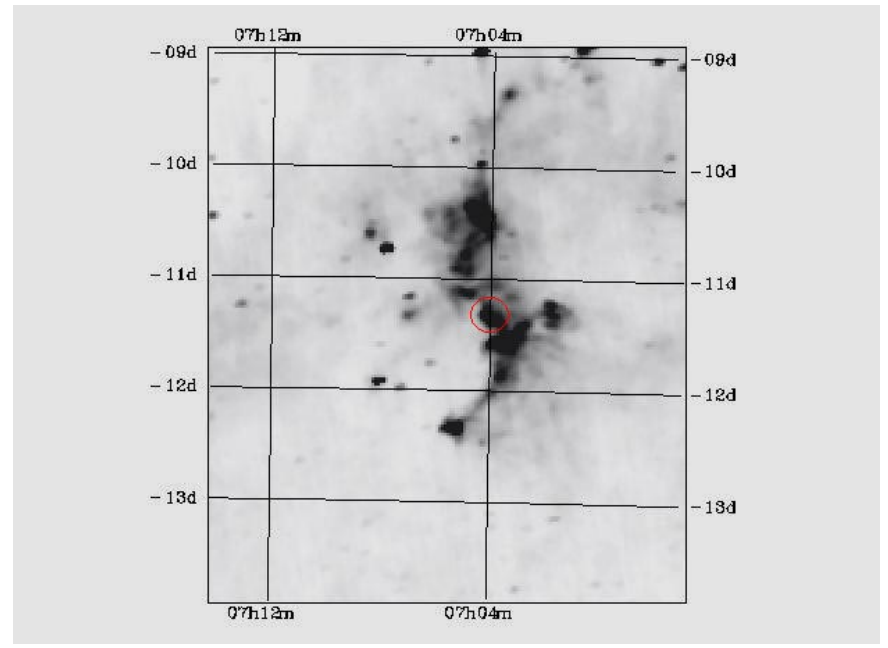

Fig. 4. IRAS $12 \mu \mathrm{m}$ image of CMaR1. Open circle encompasses BRC 27 and NGC 2327.

Table 1. Position and number of stars for 2MASS extractions.

\begin{tabular}{lccc}
\hline \hline Name & $\alpha(\mathrm{J} 2000)$ & $\delta(\mathrm{J} 2000)$ & $n^{a}\left(r=1^{\prime}\right)$ \\
\hline BRC 27 & $7^{\mathrm{h}} 04^{\mathrm{m}} 04^{\mathrm{s}}$ & $-11^{\circ} 23^{\prime} 40^{\prime \prime}$ & 29 \\
NGC 2327 & $7^{\mathrm{h}} 04^{\mathrm{m}} 08^{\mathrm{s}}$ & $-11^{\circ} 18^{\prime} 57^{\prime \prime}$ & 21 \\
Field 1 & $7^{\mathrm{h}} 03^{\mathrm{m}} 47^{\mathrm{s}}$ & $-11^{\circ} 16^{\prime} 50^{\prime \prime}$ & 7 \\
Field 2 & $7^{\mathrm{h}} 04^{\mathrm{m}} 26^{\mathrm{s}}$ & $-11^{\circ} 17^{\prime} 31^{\prime \prime}$ & 18 \\
Field 3 & $7^{\mathrm{h}} 03^{\mathrm{m}} 43^{\mathrm{s}}$ & $-11^{\circ} 31^{\prime} 28^{\prime \prime}$ & 6 \\
Field 4 & $7^{\mathrm{h}} 04^{\mathrm{m}} 27^{\mathrm{s}}$ & $-11^{\circ} 31^{\prime} 07^{\prime \prime}$ & 14 \\
\hline
\end{tabular}

${ }^{a}$ A cutoff $J=16$ was used.

corresponding colours, the respective errors, and J2000.0 coordinates.

In Fig. 5 we illustrated the 2MASS photometry errors in each magnitude for an extraction of radius $2^{\prime}$ centered on $\mathrm{LkH} \alpha 221$. Basically, the errors become important for magnitudes fainter than $K_{\mathrm{s}}=14$. We applied a cutoff at $K_{\mathrm{s}}=14.5$ for the stars to be studied in colour-colour diagrams, for the sake of accuracy. For comparison purposes we made extractions for the objects and their surrounding fields. Typical separations for objects and fields are $\approx 5^{\prime}$. Table 1 condenses information on the extractions. We show positions of the objects and fields (fields are shared in the analyses of the objects), and numbers of extracted stars (with a cutoff at $J=16$ ) for radii $1^{\prime}$. The numbers of stars in the cluster extractions exceed those of the fields.

\section{The analysis method: The Trapezium cluster as template}

Clusters in regions of large dust concentration probably have stars at the PMS stages, and we can use reference clusters for comparison, in particular their near-IR colourcolour diagrams.

In the following we will use the well-known Trapezium cluster as reference object for the analysis. The 2MASS

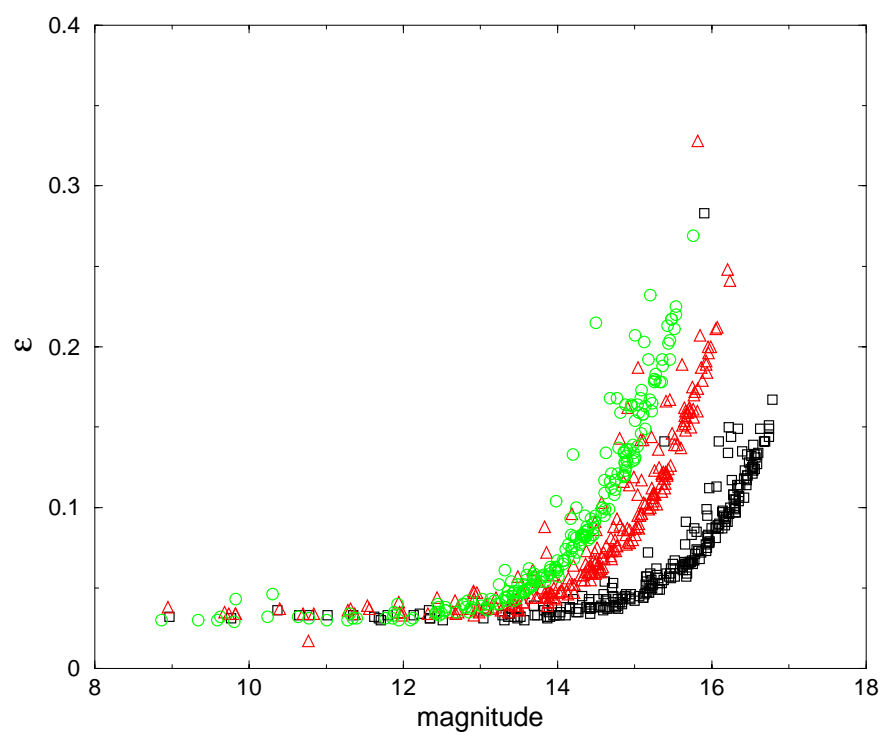

Fig. 5. 2MASS photometric errors in the bands $J$ (squares), $H$ (triangles) and $K_{\mathrm{s}}$ (circles).

photometry for Trapezium was extracted within a radius $2^{\prime}$ centered at $\alpha=5^{\mathrm{h}} 35^{\mathrm{m}} 20^{\mathrm{s}} \delta=-5^{\circ} 23^{\prime} 09^{\prime \prime}$ (J2000.0).

Both BRC 27 and NGC 2327 are dust embedded objects, probably in the stellar formation phase. Thus mainsequence isochrones (e.g. Girardi et al. 2000) are not suitable to extract information about the object parameters, since these isochrones do not include the PMS stage. One way to deal with this problem is to make use of a cluster in a comparable evolutionary stage, with known parameters. The Trapezium cluster meets these criteria, and is hereafter employed as a template cluster.

The region surrounding the Trapezium in the Orion Nebula has the largest density of young stars in our vicinity, with a peak value of $2 \times 10^{6} \mathrm{pc}^{-3}$ in the central region (Hillenbrand 1997). Age values in the range $1 \mathrm{Myr}$ (Prosser et al. 1994; Hillenbrand 1997) to 2 Myr (Palla \& Stahler 1999) have been derived.

\subsection{Colour-colour diagram}

In Fig. 6 we show the $\left((J-H),\left(H-K_{\mathrm{s}}\right)\right)$ colour-colour diagram of the Trapezium cluster. The continuous curve represents the intrinsic colours of main-sequence (MS) stars with spectral types from O3 to M5 (Schmidt-Kaler 1982). The dashed line denotes the reddening law (Rieke \& Lebofsky 1985) for a MS O3 star, while the point-dashed line is for an M5 giant. The arrow indicates the modulus, direction and sense of the extinction vector. We applied a 2MASS sample cutoff at $K_{\mathrm{s}}=14.5$ in order to avoid errors larger than $0.1 \mathrm{mag}$.

From the data of Rieke \& Lebofsky (1985) we obtained the slope of the reddening vector in the colour-colour diagrams:

$\frac{E(J-H)}{E\left(H-K_{\mathrm{s}}\right)}=1.74$ 


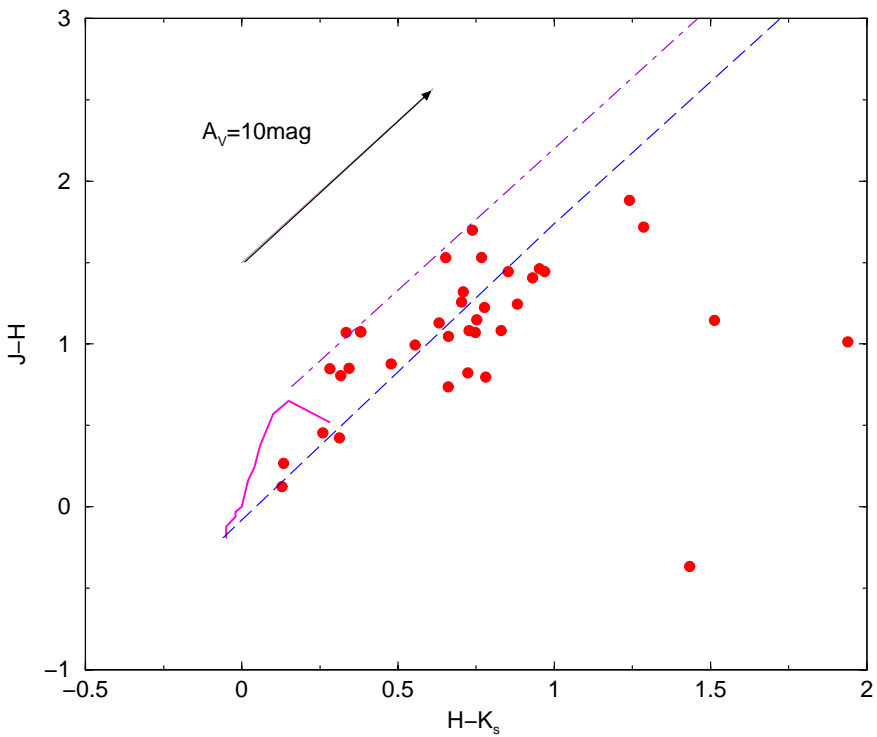

Fig. 6. Trapezium: colour-colour diagram $\left((J-H),\left(H-K_{\mathrm{s}}\right)\right)$. Continuous curve represents the intrinsic colours of MS stars with spectral types from O3 (left end) to M 5. Dashed line denotes the reddening law for a MS O3 star, while point-dashed line for an M 5 giant. Arrow indicates a reddening vector. A sample cutoff at $K_{\mathrm{s}}=14.5$ was applied to minimize errors.

Figure 6 shows a maximum stellar absorption $A_{V} \approx 10$ for the observed stars in the Trapezium, discarding the stars with infrared excess, i.e., those outside the strip along which normal stars can be reddened. The stars with anomalous colours are probably objects at the formation stage. T Tauri and Herbig AeBe stars can exhibit such anomalous colours. The $K_{\mathrm{s}}$ band excess is due to hot dust emission found around them (Lada \& Adams 1992; Calvet et al. 1992). Some T Tauri and Herbig AeBe stars do not necessarily present an anomalous colour (Lada \& Adams 1992). The fraction of sources with $K_{\mathrm{s}}$ excess in a young stellar cluster or group appears to depend on the evolutionary stage. For example, $50 \%$ to $70 \%$ of the dust embedded stars in the $\rho$ Ophiuchi Dark Nebula have infrared excess (Greene \& Young 1992; Strom et al. 1995). Recently, spectroscopic observations of stars in this cloud provided an age of 1 Myr or less (Greene \& Meyer 1995). Similarly, nearly $50 \%$ of the stars in the Taurus Dark Nebula exhibit infrared excess and an age of $1.5 \mathrm{Myr}$ is estimated (Kenyon \& Hartmann 1995). On the other hand, only $20 \%$ of the members of IC 348 with age 5-7 Myr present infrared excess (Lada \& Lada 1995). The duration of the dust circumstellar disk is estimated to be 1-3 Myr (Strom et al. 1995). This fact indicates that the stellar fraction with infrared excess decreases with the time. Therefore, the presence of stars with $K_{\mathrm{s}}$ excess is an age indicator, useful for dust embedded clusters. In the particular case of the Trapezium cluster, the percentage of anomalous stars is about $60 \%$, suggesting that the cluster has an age $\approx 1 \mathrm{Myr}$, likewise the stars in the $\rho$ Ophiuchi Dark Nebula.

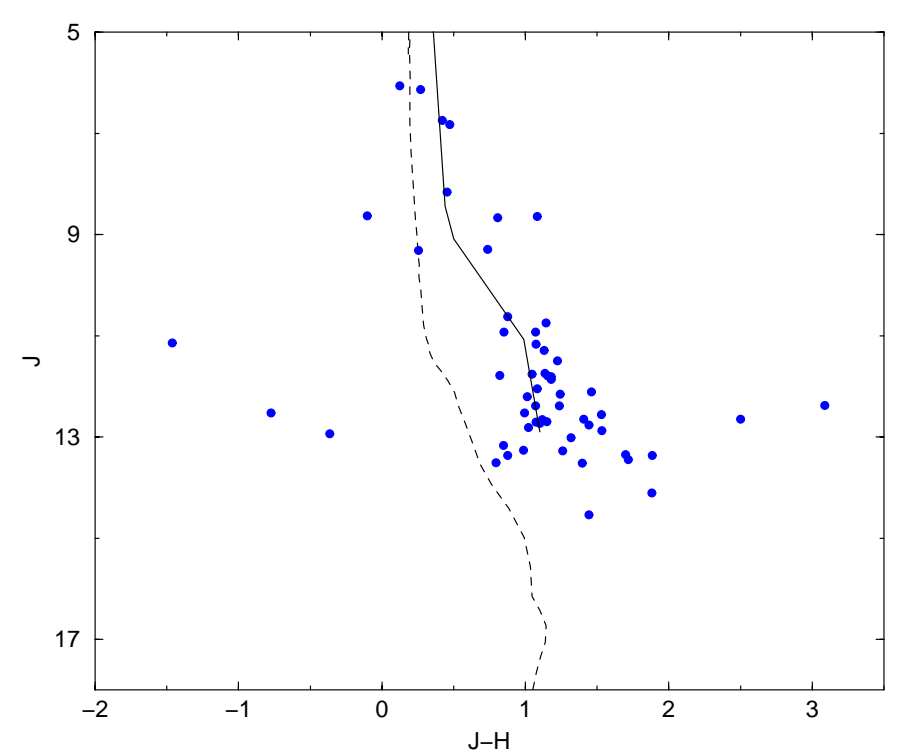

Fig. 7. Trapezium: $J,(J-H)$ CMD. Continuous line is mean locus of Trapezium, and dashed line is theoretical isochrone of $4 \mathrm{Myr}$.

\subsection{Distance from the infrared}

We will derive a reference sequence for the stars in the Trapezium cluster and obtain the apparent distance modulus which in turn will be useful for the parameter derivation of the sample clusters. The four bright stars that form the trapezium shape were already focus of a large number of studies and have known spectral classification, which we adopted from SIMBAD. As a first step we obtained the distance to the cluster through the spectral type of these stars.

The brightest star $\theta_{1}$ Ori $\mathrm{C}$ (HD 37022) of spectral type $\mathrm{O} 6$ is not present in the 2MASS photometry. The star $\theta_{1}$ Ori A (HD 37020) of spectral type O7 was not included owing to lack of information on the luminosity class, and variability. The calculations are based on the stars $\theta_{1}$ Ori B (HD 37021) and $\theta_{1}$ Ori D (HD 37023) which are MS stars with spectral types given in Table 2. Intrinsic magnitudes and colours are from Schmidt-Kaler (1982) and Knoedlselder (2000). We derived the apparent distance modulus and distance as given in Table 2, using the relation $A_{J}=2.73 E(J-H)$. The latter relation was derived from the data in Rieke and Lebofsky (1985) assuming $A_{V}=3.2 E(B-V)$. The mean apparent distance modulus is $\left(J-M_{J}\right)=9.40 \pm 0.39$, and the mean distance is $d_{\odot}=0.52 \mathrm{kpc}$, in agreement with distance estimates to the Orion Complex (Warren \& Hesser 1978).

Using the available stars in the Trapezium and the concentration of PMS stars as constraints we traced a besteye fit curve to be used as reference sequence for sample objects (Sects. 5 and 6). This reference sequence is shown in Fig. 7, together with a 4 Myr Padova isochrone for comparison purposes. The latter isochrone locus has used as constraint the MS stars. The massive stars certainly produce cavities owing to the ultraviolet flux, decreasing the dust columns. This effect can produce a differential 
Table 2. Trapezium bright stars.

\begin{tabular}{lccccccccc}
\hline \hline Star & ST & $M_{J}$ & $(J-H)_{0}$ & $J$ & $(J-H)$ & $E(J-H)$ & $A_{J}$ & $\left(J-M_{J}\right)$ & $d_{\odot}$ \\
\hline$\theta_{1}$ Ori B & B0 V & -3.63 & -0.12 & 6.13 & 0.27 & 0.39 & 1.07 & 9.76 & $0.55 \mathrm{kpc}$ \\
$\theta_{1}$ Ori D & B0.5 V & -2.98 & -0.11 & 6.06 & 0.12 & 0.23 & 0.63 & 9.04 & $0.48 \mathrm{kpc}$ \\
\hline
\end{tabular}

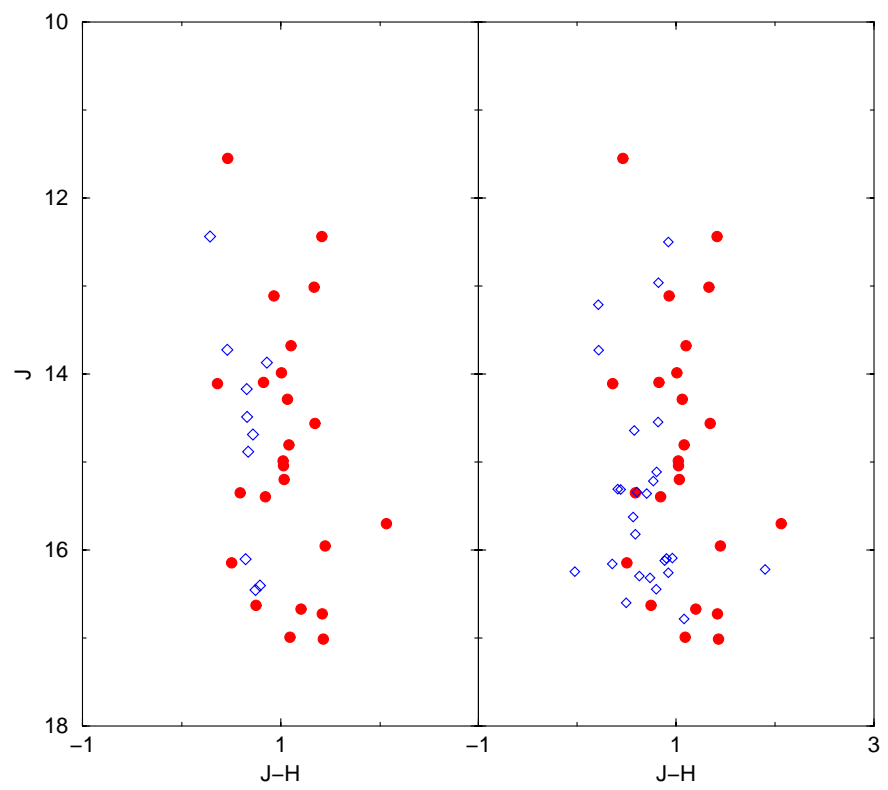

Fig. 8. NGC 2327: $J,(J-H)$ CMD of the cluster region (filled circles) and comparison star fields (open diamonds). Field 1 corresponds to the left panel and field 4 to the right one. All extractions have radius $1^{\prime}$. No magnitude cutoff was used.

reddening with respect to low mass stars. However such possible reddening differences will be implicit in the reference sequence.

\section{The cluster in the nebula NGC 2327}

Dust rich regions can be associated to young star clusters, in general related with the stellar contraction stage. These regions are better explored in IR bands. In Fig. 8 we show the $J,(J-H)$ CMD for an extraction of radius $1^{\prime}$, centered on NGC 2327. We show equal size extractions for the comparison fields 1 and 4 (Table 1 ). It is clear the redder colours for the stars in the cluster region, suggesting larger reddening. Field 4 contains more stars than field 1 possibly owing to the larger distance from the nebula and less dust mixed to the stars.

Figure 9 shows a $\left((J-H),\left(H-K_{\mathrm{s}}\right)\right)$ colour-colour diagram for the NGC 2327 cluster, compared to that of field 4. A significant number of stars in the cluster present infrared excess $\left(H-K_{s}>1\right)$. The remaining cluster stars present a larger reddening than those of the field.

The largest reddening in Fig. 9 is $A_{V} \approx 11$. A calculated mean value resulted $A_{V}=5.5$. For this estimation we excluded the stars with infrared excess and those superposed on the field locus.

We can estimate the cluster age by means of the percentage of cluster stars with infrared excess (Sect. 4).

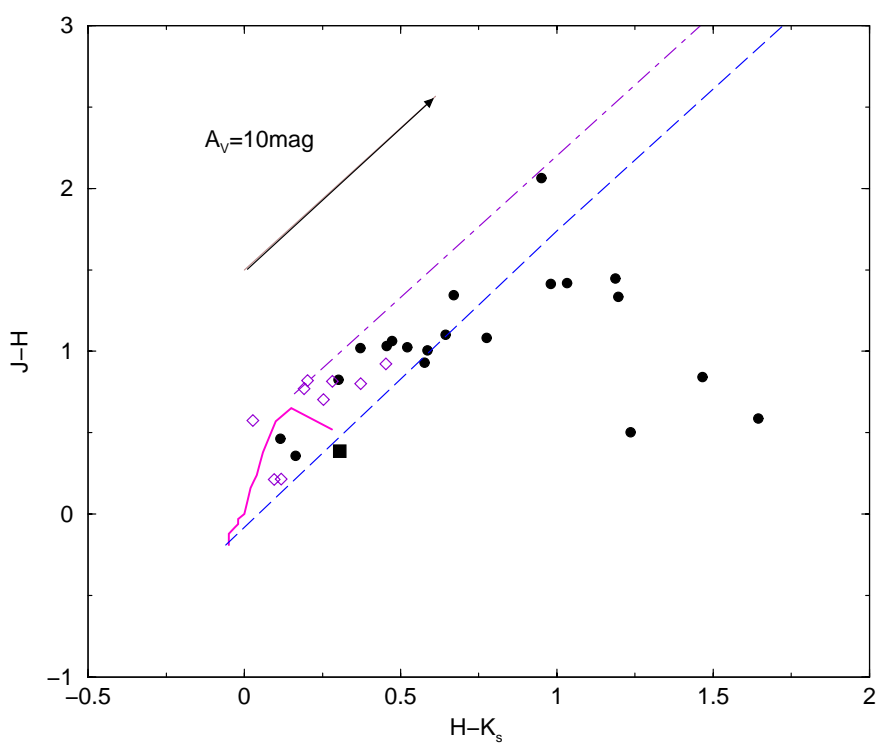

Fig. 9. NGC 2327: colour-colour diagram $\left((J-H),\left(H-K_{\mathrm{s}}\right)\right)$ for the cluster (filled circles) and comparison field 4 (open diamonds). Filled square is $\mathrm{LkH} \alpha 221$. All extractions are for radius $r<1^{\prime}$. Reference lines as in Fig. 6 . A cutoff $K_{\mathrm{s}}=14.5$ was applied.

In order to minimize the field contamination we discarded the stars with extinction similar to those of field stars. The NGC 2327 cluster has $\approx 50 \%$ of stars with infrared excess, implying that the object has an age of about $1.5 \mathrm{Myr}$ (Sect. 4.1).

By matching the Trapezium sequences to those of the NGC 2327 cluster (Fig. 10) we obtain $\Delta J=J_{\text {Trap }}$ $J_{\mathrm{NGC} 2327}=2.4$. Considering the Trapezium apparent distance modulus (Sect. 4.3) we derive for the NGC 2327 cluster $\left(J-M_{J}\right)=11.8 \pm 0.3$. The apparent distance modulus uncertainty in the template itself (Sect. 4.2) leads to a total error $\tau=0.49$. We adopted the reddening from the colour-colour diagram (Fig. 9) which implies $A_{J}=1.5$. In the latter calculation we transformed $V$ to $J$ absorption, using the relation $\frac{A_{J}}{A_{V}}=0.276$ (Schlegel et al. 1998). We found a distance from the Sun $d_{\odot}=1.15 \pm 0.26 \mathrm{kpc}$.

The brightest star in the direction of NGC 2327 is $\mathrm{LkH} \alpha 221\left(\alpha=7^{\mathrm{h}} 04^{\mathrm{m}} 06^{\mathrm{s}} \delta=-11^{\circ} 18^{\prime} 34^{\prime \prime}(\mathrm{J} 2000)\right)$ with spectral type B5 (Clariá 1974a). The 2MASS magnitude is $J=9.66$ and colours are $(J-H)=0.39$ and $\left(H-K_{\mathrm{s}}\right)=0.31$ for this star. A B5 dwarf has $M_{J}=-0.82$ and $(J-H)_{0}=-0.06$ (Schmidt-Kaler 1982) which imply for $\mathrm{LkH} \alpha 221 A_{J}=1.16$, an apparent distance modulus $\left(J-M_{J}\right)=10.5$ and a distance $d_{\odot}=0.72 \mathrm{kpc}$. The resulting distance is too small as compared to that derived for the cluster, certainly because $\mathrm{LkH} \alpha 221$ has a K0 T Tauri component (Cohen \& Kuhi 1979). Note also 


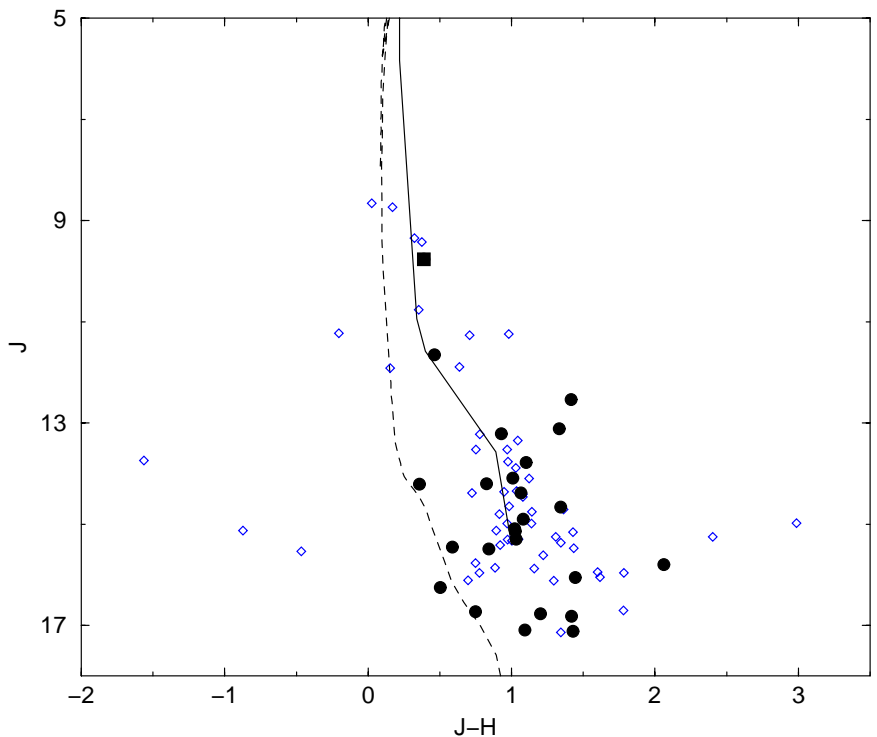

Fig. 10. NGC 2327: $J,(J-H)$ CMD of the cluster (filled circles) with reference line from Trapezium (continuous line). Filled square is $\mathrm{LkH} \alpha 221$. Padova isochrone for $4 \mathrm{Myr}$ (dashed line) is shown for comparison. Trapezium stars are also shown (open diamonds).

that in Fig. 9 the star has a small infrared excess. We conclude that these effects explain this distance discrepancy as estimated from the infrared.

\section{The cluster in the nebula BRC 27}

In Fig. 11 we show CMDs $(J, J-H)$ for the cluster and the comparison fields 1 and 4. Likewise in NGC 2327 (Sect. 5) the stars in the cluster region are redder than those in the fields, denoting the important dust column in this embedded cluster direction.

The colour-colour diagram (Fig. 12) presents a number of stars distributed along the reddening vector, and some are little reddened $\left(A_{V} \approx 2\right)$ and coincident with the field locus. The strongest reddening corresponds to $A_{V} \approx 15$. The mean reddening is $A_{V}=6.5$ considering only the stars along the reddening vector and non coincident with those of the field. Several stars have $\left(H-K_{\mathrm{s}}\right)$ colours denoting infrared excess. The fraction of these stars with respect to all sampled cluster stars is about $50 \%$, as in the case of the NGC 2327 cluster (Sect. 5), implying an age $\approx 1.5$ Myr for the BRC 27 cluster.

In Fig. 13 is shown the same CMD of the BRC 27 cluster as in Fig. 11 where we matched the Trapezium sequences. No massive star was formed in BRC 27. We obtained $\Delta J=J_{\text {Trap }}-J_{\mathrm{BRC} 27}=2.8$. Considering the Trapezium apparent distance modulus (Sect. 4.3) we derived for the BRC 27 cluster $\left(J-M_{J}\right)=12.2 \pm 0.3$. The total error is $\tau=0.51$ (Sect. 4.1). By adopting the colourcolour diagram reddening (Fig. 12) we found $A_{J}=1.8$. We obtained a distance from the Sun $d_{\odot}=1.20 \pm 0.27 \mathrm{kpc}$.

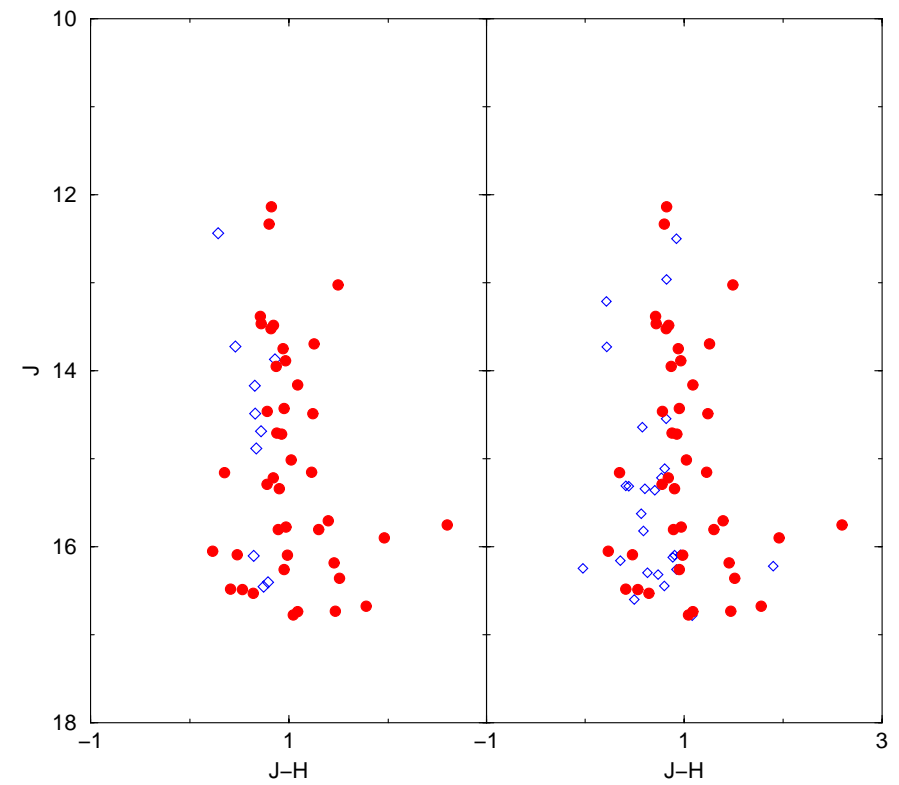

Fig. 11. BRC 27: $J,(J-H)$ CMD of the cluster region (filled circles) and comparison star fields (open diamonds). Field 1 corresponds to the left panel and field 4 to the right one. All extractions have radius $1^{\prime}$. No cutoff was used.

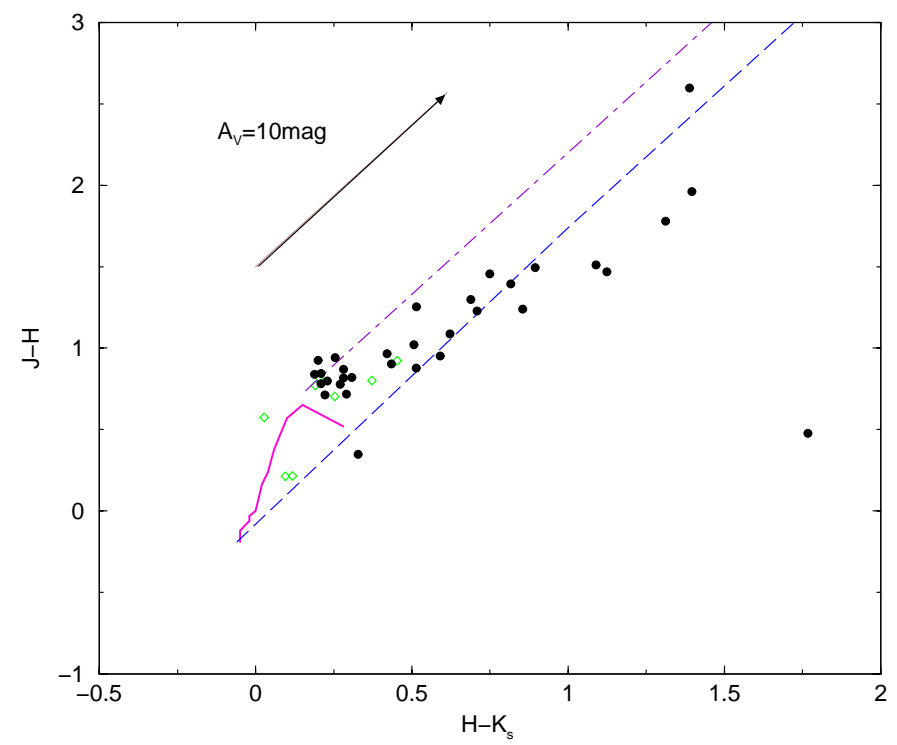

Fig. 12. BRC 27: colour-colour diagram $\left((J-H),\left(H-K_{\mathrm{s}}\right)\right)$ for the cluster (filled circles) and comparison field 4 (open diamonds). Extractions correspond to radius $<1^{\prime}$. Reference lines as in Fig. 6. A cutoff at $K_{\mathrm{s}}=14.5$ was used.

\section{Concluding remarks}

Two embedded star clusters in CMaR1 were studied by means of 2MASS $J H K_{\mathrm{S}}$ photometry, respectively in the nebula NGC 2327 and in the bright-rimmed cloud BRC 27.

The NGC 2327 cluster is studied for the first time. It has $\approx 50 \%$ of its stars with infrared excess, implying an age of about 1.5 Myr, comparable to that of the Trapezium cluster and the star-forming event in the Taurus Dark Nebula (Kenyon \& Hartmann 1995). The mean visible absorption in NGC 2327 is $A_{V}=5.5$ and in BRC 27 is 


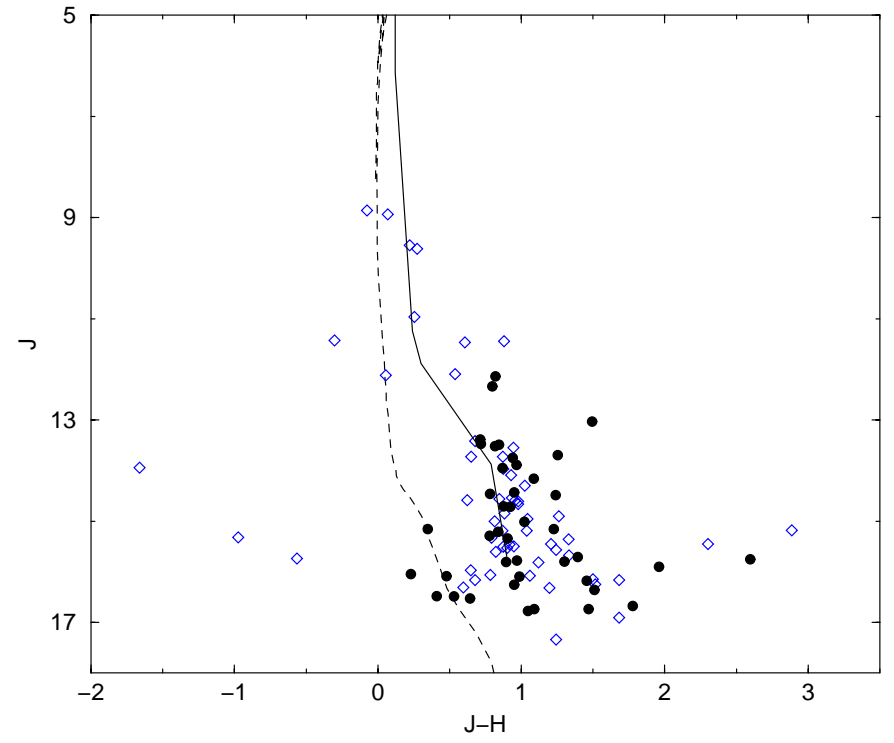

Fig. 13. BRC 27: $J,(J-H)$ CMD of the cluster (filled circles) with reference line from Trapezium (continuous line). Padova isochrone for $4 \mathrm{Myr}$ (dashed line) is shown for comparison. Trapezium stars are also shown (open diamonds).

$A_{V}=6.5$, as derived from the colour-colour diagram. The BRC 27 cluster colour-magnitude diagram is similar to that of the NGC 2327 cluster indicating that both are in a comparable evolutionary stage. Distances were estimated via comparison with the mean stellar evolutionary sequences of the Trapezium cluster. Both sample clusters result at a distance from the Sun $d_{\odot} \approx 1.2 \mathrm{kpc}$, comparable within uncertainties to previous estimates of the CMaOB1/CMaR1 distance (Clariá 1974b; Shevchenko 1999). Some embedded star clusters are double, such as those in the NGC 1333 region (Lada et al. 1996), which are separated by $\approx 1$ diameter. The coeval nature, distance and projected separation of $\approx 2.5$ diameters suggest the birth of a physical pair of clusters in CMaR1, from the same star-forming event (Fig. 4).

Acknowledgements. We thank Daniela B. Pavani for discussions. We thank the referee Prof. Seggewiss for interesting remarks. This publication makes use of data from the 2 Micron All Sky Survey, which is a joint project of the
University of Massachusetts and the Infrared Processing and Analysis Center, funded by the National Aeronautics and Space Administration and the National Science Foundation. We employed data from the CDS database (Strasbourg). We also acknowledge support from the Brazilian Institutions $\mathrm{CNPq}$ and CAPES, in particular J.S. for a $\mathrm{CNPq} \mathrm{PhD}$ fellowship.

\section{References}

Calvet, N., Magris, G. C., \& Patino, A. 1992, RMxAA, 24, 27 Carpenter, J. M., Snell, R. L., Schloerb, F. P., \& Skrutskie, M. F. 1993, ApJ, 407, 657

Clariá, J. J. 1974a, ApJ, 79, 1022

Clariá, J. J. 1974b, A\&A, 37, 229

Cohen, M., \& Kuhi, L. V. 1979, ApJS, 41, 743

Girardi, L., Bressan, A., Bertelli, G., \& Chiosi, C. 2000, A\&AS, 141, 371

Greene, T. P., \& Meyer, M. 1995, ApJ, 450, 233

Greene, T. P., \& Young, E. T. 1992, ApJ, 249, 481

Hillenbrand, L. A. 1997, AJ, 113, 1733

Hodapp, W. K. 1994, ApJ, 94, 615

Kenyon, S. J., \& Hartmann, L. 1995, ApJS, 101, 117

Knoedlselder, J. 2000, A\&A, 360, 539

Lada, C. J., \& Adams, F. 1992, ApJ, 393, 278

Lada, E. A., \& Lada, C. J. 1995, AJ, 109, 1682

Lada, C. J., Alves, J., \& Lada, E. A. 1996, AJ, 111, 1964

Palla, F., \& Stahler, S. W. 1999, ApJ, 525, 772

Prosser, C. F., Stauffer, J. R., Hartmann, L., et al. 1994, ApJ, 421,517

Rieke, G. H., \& Lebofsky, M. J. 1985, ApJ, 288, 618

Schlegel, D. J., Finkbeiner, D. P., \& Davis, M. 1998, ApJ, 500, 525

Schmidt-Kaler, T. 1982, in Landolt-Börnstein, New Ser., Group VI, vol. 2b (Springer-Verlag, Berlin), 1

Shevchenko, V. S. 1999, MNRAS, 310, 210

Skrutskie, M., Schneider, S. E., Stiening, R., et al. 1997, in The Impact of Large Scale Near-IR Sky Surveys, ed. F. Garzon et al. (Dordrecht: Kluwer), 25

Strom, K. M., Kepner, J., \& Strom, S. E. 1995, ApJ, 438, 8130

Sugitani, K., Fukui, Y., \& Ogura, K. 1991, ApJS, 77, 59

Sugitani, K., Tamura, M., \& Ogura, K. 1995, ApJ, 455, L39

van den Bergh, S. 1966, AJ, 71, 990

Warren, W. H., Jr., \& Hesser, J. E. 1978, ApJS, 36, 497

Weintraub, D. A. 1990, ApJS, 74, 575 